\title{
Photoproduction of vector mesons: from ultraperipheral to semi-central heavy ion collisions
}

\author{
Mariola Kłusek-Gawenda ${ }^{1, \star}$ and Antoni Szczurek ${ }^{1,2}$ \\ ${ }^{1}$ Institute of Nuclear Physics, Polish Academy of Sciences, Kraków, Poland \\ ${ }^{2}$ University of Rzeszów, Rzeszów, Poland
}

\begin{abstract}
We discuss nuclear cross sections for $A A \rightarrow A A V$ and $A A \rightarrow A A V V$ reactions with one or two vector mesons in the final state. Our analysis is done in the impact parameter space equivalent photon approximation. This approach allows to consider the above processes taking into account distance between colliding nuclei. We consider both ultraperipheral and semi-central collisions. We are a first group which undertook a study of single $J / \psi$ photoproduction for different centrality bins. We show that one can describe new ALICE experimental data by including geometrical effects of collisions in the flux factor.

Next, total and differential cross section for double-scattering mechanism in the exclusive $A A \rightarrow A A V V$ reaction in ultrarelativistic ultraperipheral heavy ion collisions is presented. In this context we consider double photoproduction and photon-photon processes. Simultaneously, we get very good agreement of our results with STAR (RHIC), CMS and ALICE (LHC) experimental data for single $\rho^{0}$ and $J / \psi$ vector meson production. A comparison of our predictions for exclusive four charged pions production is also presented.
\end{abstract}

\section{Introduction}

In last years there was an interest in calculating cross sections for exclusive production of vector mesons in ultrarelativistic heavy ion collisions [1-10]. Purely ultraperipheral collisions (UPC) of heavy ions mean the situation when the nuclei do not collide, however, an emission of extra neutrons caused by additional purely electromagnetic interactions could be included. In theoretical calculations UPC means in practice $b>R_{A}+R_{B}$ (sum of nuclear radii). All the calculations must be therefore performed in the impact parameter space to include this condition.

We discuss an importance of photoproduction process in the context of $J / \psi$ production in less peripheral collisions. Then, at high energies, nuclei can collide and break apart producing quarkgluon plasma. At low transverse momenta and small multiplicities the ALICE Collaboration [11] observed an enhancement of nuclear modification factor at small transverse momenta $R_{A A}>1$.

Nuclear cross sections are calculated with the help of equivalent photon approximation (EPA) in the impact parameter space. We include realistic form factor of nuclei which is Fourier transform of the charge distribution in nucleus. The differences of the cross sections with realistic and monopole form factor can be found e.g. in Ref. [12].

\footnotetext{
^e-mail: mariola.klusek@ifj.edu.pl,
} 


\section{Some theoretical aspect}

Fig. 1 depicts the difference between ultraperipheral and semi-central collisions of heavy ions. The left panel shows UPC for production of vector mesons. The two-dimensional vector $\vec{b}$ is a distance between colliding nuclei, $\overrightarrow{b_{1}}$ is a distance between the photon position and the middle of the first (emitter) nucleus and $\overrightarrow{b_{2}}$ is a distance between the photon position and the middle of the second (medium) nucleus. The production of the vector meson may occur provided the photon (hadronic fluctuation) hits the second nucleus. The hatched area of overlapping nuclei in the right panel (semicentral collision) of the figure represents the area in the impact parameter space for which some absorption may be expected. We include the effect of the "absorption" by modifying effective photon fluxes in the impact parameter space by imposing several additional geometrical conditions on impact parameters (between photon and nuclei and/or between colliding nuclei).
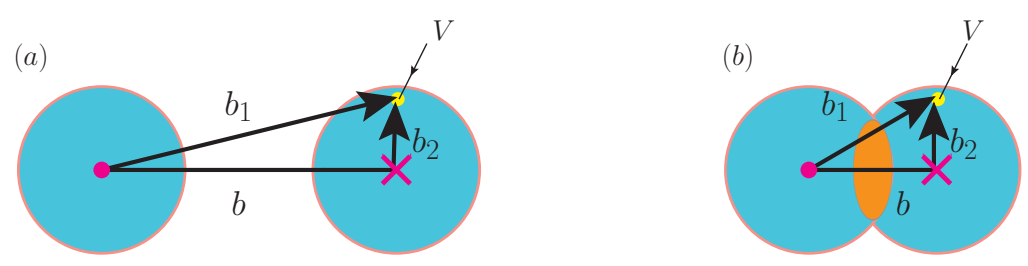

Figure 1. Impact parameter picture of the collision and the production of the vector meson for ultraperipheral (left panel) and for semi-central (right panel) collisions. It is assumed here that the first nucleus is the emitter of the photon which rescatters then in the second nucleus being a rescattering medium.

The left panel of Fig. 2 shows a single vector meson production mechanism. Photon emitted from a nucleus fluctuates into hadronic or quark-antiquark components and converts into an on-shell meson. The differential cross section for this mechanism can be written as

$$
\frac{\mathrm{d} \sigma_{A_{1} A_{2} \rightarrow A_{1} A_{2} V}}{\mathrm{~d}^{2} b \mathrm{~d} y}=\frac{\mathrm{d} P_{\gamma \mathbf{P}}(b, y)}{\mathrm{d} y}+\frac{\mathrm{d} P_{\mathbf{P} \gamma}(b, y)}{\mathrm{d} y} .
$$

$P_{\gamma \mathbf{P}}(y, b) / P_{\mathbf{P} \gamma}(y, b)$ are the probability densities for producing a vector meson at rapidity $y$ for fixed impact parameter $b$ (distance between two colliding nuclei) of the heavy ion collision. Each probability is the convolution of a flux of equivalent photon and the $\gamma A \rightarrow V A$ cross section.
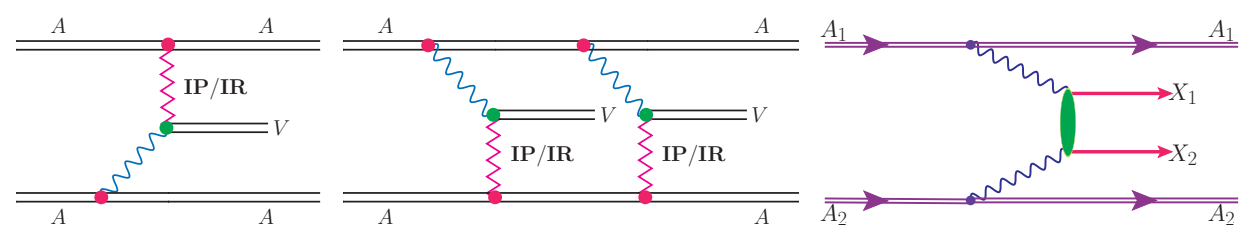

Figure 2. Single vector meson production by photon-Pomeron (or Pomeron-photon) fusion (left panel) and double vector meson production for double-scattering mechanism (middle panel) and for $\gamma \gamma$ fusion (right panel).

The middle panel of Fig. 2 illustrates one of four mechanisms of the double-scattering mechanism (in fact, we take into account all four combinations of $\gamma \mathbb{P}$ exchanges: $\gamma \mathbb{P}-\gamma \mathbf{P}, \gamma \mathbb{P}-\mathbf{P} \gamma, \mathbb{P} \gamma-\gamma \mathbb{P}$, $\mathbb{P} \gamma-\mathbb{P} \gamma)$. Having a formalism for the calculation of single-vector-meson production, one can use 
it to calculate cross section for double-scattering mechanisms of two-vector-meson production. The cross section for the double vector meson photoproduction is expressed with the help of probability density of single meson production

$$
\frac{\mathrm{d} \sigma_{A_{1} A_{2} \rightarrow A_{1} A_{2} V V}}{\mathrm{~d} y_{1} \mathrm{~d} y_{2}}=\frac{1}{2} \int \mathrm{d}^{2} b\left[\left(\frac{\mathrm{d} P_{\gamma \mathbf{P}}\left(b, y_{1}\right)}{\mathrm{d} y_{1}}+\frac{\mathrm{d} P_{\mathbf{P} \gamma}\left(b, y_{1}\right)}{\mathrm{d} y_{1}}\right) \times\left(\frac{\mathrm{d} P_{\gamma \mathbf{P}}\left(b, y_{2}\right)}{\mathrm{d} y_{2}}+\frac{\mathrm{d} P_{\mathbb{P} \gamma}\left(b, y_{2}\right)}{\mathrm{d} y_{2}}\right)\right] .
$$

For completeness, in the right panel of Fig.2, we show Feynman diagram for pair of vector meson production in the $\gamma \gamma$ fusion. A formula for the total cross section for this mechanism can be written in a somewhat simplified way as

$$
\sigma_{A_{1} A_{2} \rightarrow A_{1} A_{2} V V}=\int \mathrm{d} \omega_{1} \mathrm{~d} \omega_{2} n\left(\omega_{1}\right) n\left(\omega_{2}\right) \sigma_{\gamma \gamma \rightarrow V V},
$$

where $\omega_{1 / 2}$ is the photon energy from first/second nucleus. More details referring to Eq. 3 can be found in Ref. [8].

\section{Theoretical results versus experimental data}
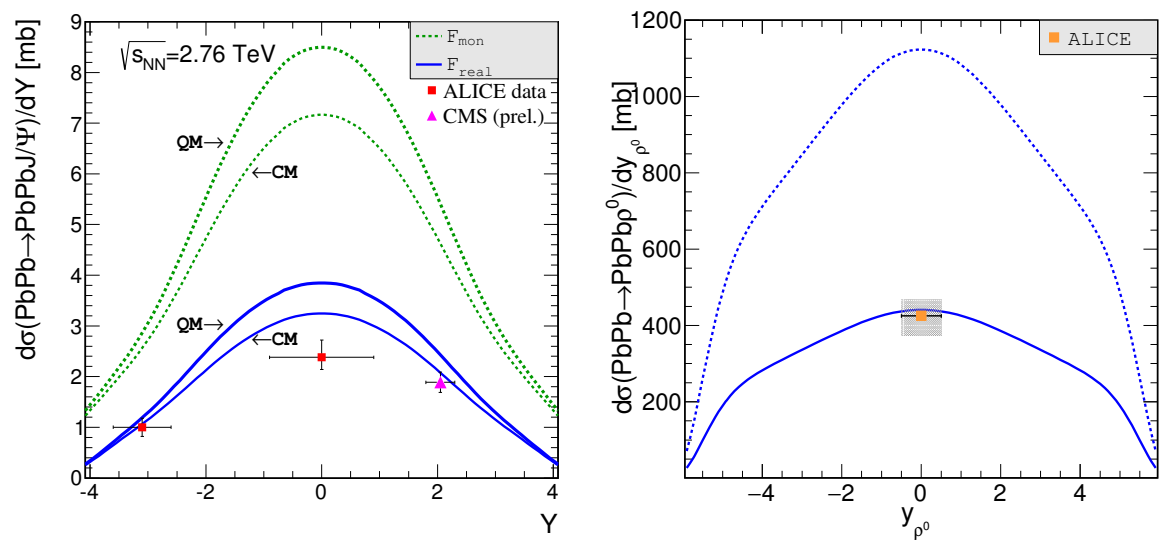

Figure 3. Left panel - differential cross section for coherent production of $J / \psi$ meson in UPC as a function of rapidity of the $J / \psi$ meson compared with the ALICE and CMS data points. Results are shown for both realistic and monopole form factor. Right panel - differential cross section for single $\rho^{0}$ photoproduction in UPC as a function of meson rapidity together with the ALICE experimental data point.

We start from purely ultraperipheral collisions. Fig. 3 shows results for coherent $J / \psi$ (left panel) and $\rho^{0}$ (right panel) photoproduction in the lead-lead UPC at the LHC energy. We show our results for classical and quantal rescattering (more details in Ref. [10]) in the nucleus-medium and for realistic and monopole nuclear form factors. The result with the monopole form factor overestimates the ALICE [14, 15] and CMS [13] data. The result with the quantal rescattering is a little larger than that for the classical rescattering. However, this difference is much smaller for $J / \psi$ production than for the photoproduction of $\rho^{0}$ meson. the ALICE data point (Ref. [16]) for $\rho^{0}$ meson production (right panel) is much better described by the classical rescattering calculations (lower line). 
Fig. 4 shows differential cross section for the $A A \rightarrow A A \rho^{0} \rho^{0}$ reaction as a function of one $\rho^{0}$ meson rapidity and a comparison of the double-scattering and $\gamma \gamma$ fusion contributions at RHIC (left panel) and at LHC (right panel) energies. The double-scattering component dominates over the $\gamma \gamma$ one. In addition, the distribution in rapidity for the RHIC energy is much narrower than that for LHC energy. The high-energy (VDM-Regge) component of the elementary cross section dominates over the low-energy bump contribution at the LHC energy. This is caused by the fact that at the higher center-of-mass energy the higher values of two-meson invariant mass becomes more important which corresponds to larger values of particle rapidity. Both at the RHIC and LHC energy, the contribution of the $\gamma \gamma$ fusion is one order of magnitude smaller than that of the double-scattering mechanism.
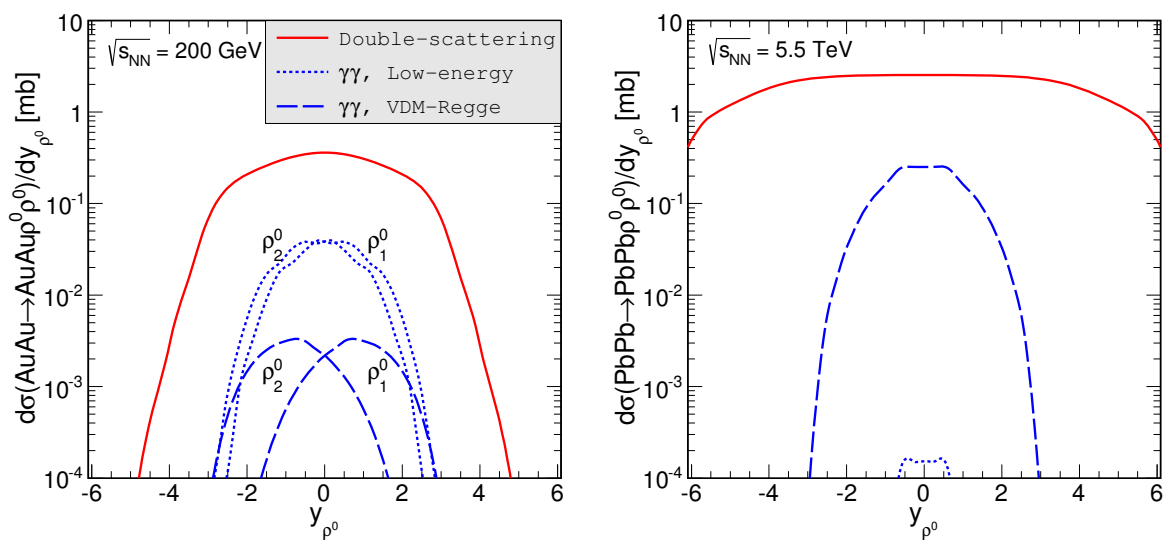

Figure 4. Rapidity distribution of one of the $\rho^{0}$ meson produced in the double-scattering mechanism and in the $\gamma \gamma$ fusion at the RHIC (left panel) and at the LHC (right panel) energy.
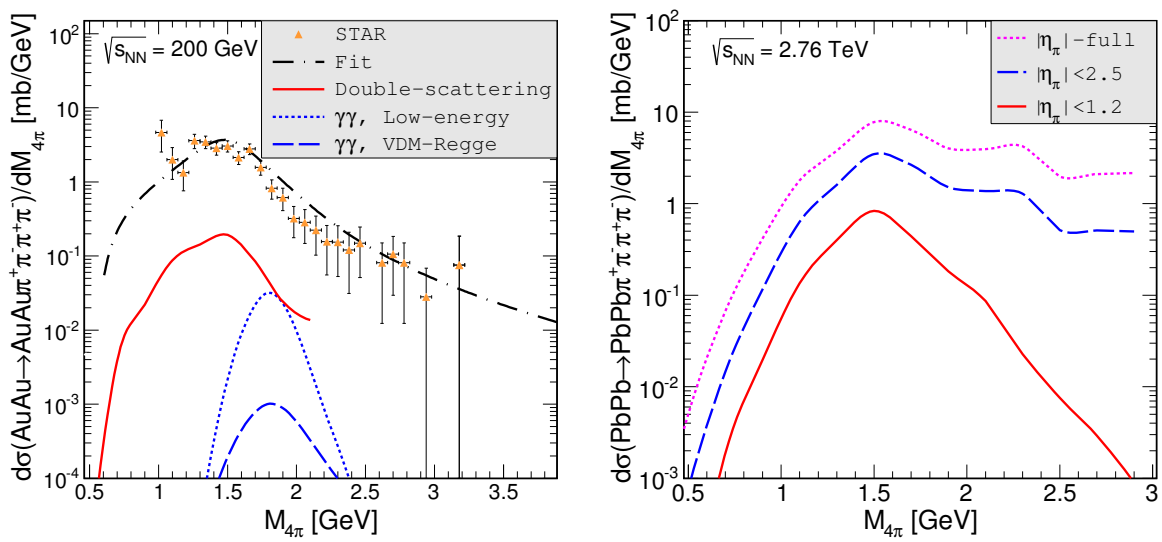

Figure 5. Invariant mass of four charged pions produced in the double-scattering mechanism and in the $\gamma \gamma$ fusion of two $\rho^{0}$ mesons at the RHIC (left panel) and at the LHC (right panel) energy. 
As in previous figures, Fig. 5 shows contributions from double-scattering, the low-energy bump and the high-energy VDM-Regge $\gamma \gamma$ fusion contributions [6] but now as a function of four-pion invariant mass. The left panel of Fig. 5 shows results for the limited acceptance of the STAR experiment $\left(\left|\eta_{\pi}\right|<1\right)$ [17]. The dash-dotted line represents a fit of the STAR Collaboration. The double-scattering contribution accounts only for $20 \%$ of the cross section measured by the STAR Collaboration. The STAR experimental data have been corrected by experimental acceptance [17]. Wu suppose that the production of the $\rho^{0}(1450)$ or $\rho^{0}(1700)$ resonance and their subsequent decay into the four-pion final state is the dominant effect for the limited STAR acceptance. However, the production mechanism of this two broad resonances and their decay into four charged pions are not yet understood. A model production of the resonances and their decay has to be study in the future. The right panel of Fig. 5 shows four-pion invariant mass distribution for double-scattering mechanism for different limited range of pion pseudorapidity at the LHC energy. The ALICE group collected the data for four-charged-pion production with the limitation $\left|\eta_{\pi}\right|<1.2$. We cannot compare this distribution with the ALICE data, because those data are not yet absolutely normalized.

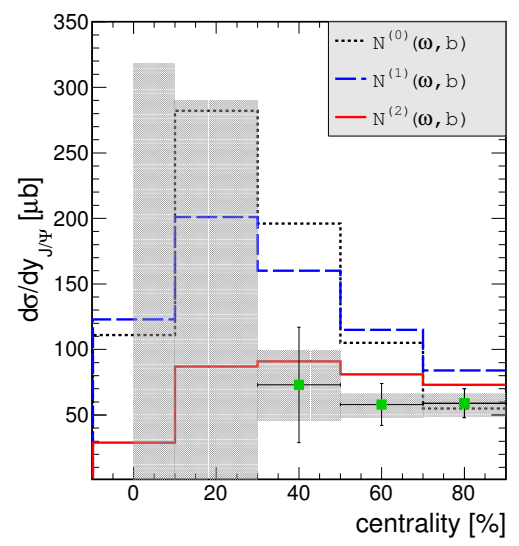

Figure 6. $d \sigma / d y$ cross sections for $\mathrm{PbPb} \rightarrow \mathrm{PbPbJ} / \psi$ reaction for different centrality bins. Theoretical results for three forms of photon fluxes [10] are compared with the ALICE data [18]. The shaded area represents the experimental uncertainties.

Going from peripheral to semi-central collisions we assume that the whole nucleus produces photons and the photon (or hadronic photon fluctuation) must hit the other nucleus to produce the vector meson. In Fig. 6 we show differential nuclear cross section for single $J / \psi$ production as a function of centrality for its small values $\left(b<R_{A}+R_{B}\right)$ i.e. for the semi-central collisions. We present new experimental ALICE data [18] with statistical and systematic error bars (shaded area). The ALICE Collaboration could not extract actual values of the cross section for the two lowest centrality bins. The results for standard photon flux (see Eq. (2.3) in Ref. [10]) exceed the ALICE data. Rather good agreement with the data is achieved for the $N^{(2)}$ photon flux (see Eq. (2.5) in Ref. [10]) obtained with the realistic nucleus form factor. In this approximation we integrate the photon flux of the first (emitter) nucleus only over this part of the second (medium) nucleus which does not collide with the nucleus-emitter (see right panel of Fig. 1). 


\section{Conclusion}

We have studied one and two vector meson production as well as four-pion production in exclusive ultrarelativistic UPC of heavy ions. In addition, we have explained theoretical transition from ultraperipheral to peripheral or semi-central cases. In our calculations we have used equivalent photon approximation in the impact parameter space. We have obtained good description of STAR and ALICE UPC data for $\rho^{0}(770)$ production and CMS and ALICE data for $J / \psi$ production. For more central nucleus-nucleus collisions we have calculated, for the first time, e.g. the differential cross section as a function of $J / \psi$ quarkonium rapidity and simultaneously we have obtained very good agreement with ALICE experimental data. We think that correct interpretation of the ALICE data suggests that the "coherent" (assumed by the formula used for the $\gamma A \rightarrow J / \psi A$ process) scattering of the hadronic fluctuation happens before the quark-gluon plasma is created. In addition, we have compared contribution of four-pion production via $\rho^{0} \rho^{0}$ production (double scattering and $\gamma \gamma$ fusion) with experimental STAR Collaboration data. The theoretical predictions have a similar shape as the distribution measured by the STAR Collaboration, but missing contribution probably come from decays of excited states of $\rho^{0}$ resonances into four charged pions.

\section{Acknowledgement}

This work was partially supported by the Polish grant No. DEC-2014/15/B/ST2/02528 (OPUS) as well as by the Centre for Innovation and Transfer of Natural Sciences and Engineering Knowledge in Rzeszów.

\section{References}

[1] L. Frankfurt, M. Strikman, M. Zhalov, Phys. Lett. B537, 51 (2002)

[2] C.A. Bertulani, S.R. Klein, J. Nystrand, Ann. Rev. Nucl. Part. Sci. 55, 271 (2005)

[3] V.P. Goncalves, M.V.T. Machado, J. Phys. G32, 295 (2006)

[4] K. Hencken, G. Baur, D. Trautmann. Phys. Rev. Lett. 96, 012303 (2006)

[5] A.J. Baltz et al., Phys. Rept. 458, 1 (2008)

[6] M. Kłusek, W. Schäfer, A. Szczurek, Phys. Lett. B674, 92 (2009)

[7] S. Baranov, A. Cisek, M. Kłusek-Gawenda, W. Schäfer, A. Szczurek, Eur. Phys. J. C73, 2335 (2013)

[8] M. Kłusek-Gawenda, A. Szczurek, Phys. Rev. C89, 024912 (2014)

[9] L. Frankfurt, V. Guzey, M. Strikman, M. Zhalov, Phys. Lett. B752, 51 (2016)

[10] M. Kłusek-Gawenda, A. Szczurek, Phys. Rev. C93, 044912 (2016)

[11] ALICE Collaboration, B. Abelev et al., Phys. Rev. Lett. 109, 072301 (2012)

[12] M. Kłusek-Gawenda, A. Szczurek, Phys. Rev. C82, 014904 (2010)

[13] CMS Collaboration, CMS-PAS-HIN-12-009 (2014) 11 pp

[14] ALICE Collaboration, B. Abelev et al., Phys. Lett. B718, 1273 (2013)

[15] ALICE Collaboration, E. Abbas et al., Eur. Phys. J. C73, 2617 (2013)

[16] ALICE Collaboration, J. Adam et al., JHEP 1509, 095 (2015)

[17] STAR Collaboration, B. Abelev et al., Phys. Rev. C81, 044901 (2010)

[18] ALICE Collaboration, J. Adam et al., Phys. Rev. Lett. 116, 222301 (2016) 